\title{
'Power-as-a-Service' - A Hierarchical On-Demand Charging Model for Recharging the Mobile Nodes of MANETs
}

\author{
https://doi.org/10.3991/ijim.v15i12.23081 \\ Muralidhar Kurni $\left({ }^{\square}\right)$ \\ Anantha Lakshmi Institute of Technology and Sciences, \\ Ananthapuram, India \\ muralidhar.kurnilieee.org \\ K. Saritha \\ S.V. Degree \& P.G. College, Ananthapuram, India
}

\begin{abstract}
Battery energy is a crucial issue that limits battery-powered mobile devices' operational efficiency in Mobile Ad hoc Networks (MANETs). Failure of a node affects both the lifetime and connectivity of a MANET, which has to initiate finding a new route from source to destination. This initiation causes more energy consumption in nodes. Failure of a node also causes network partitions, thereby resulting in sparse networks being formed. Existing energy-efficient strategies only defer the end of a node's battery lifetime; they could not guarantee the MANET's nonstop functioning. To address the issues caused by battery depletion, this paper proposes a "Cloud" oriented approach called Power-as-a-Service (PaaS), a hierarchical on-demand charging model for recharging the mobile nodes of the MANET. In PaaS, the MANET is alienated into non-overlapping disjoint zones, and for each zone, one Zone Charging Cloud Node $(\mathrm{ZccN})$ is deployed to recharge the mobile nodes of that particular zone wirelessly. A High-power Charging Cloud Node $(\mathrm{HccN})$ is deployed to wirelessly recharge the ZccNs in the MANET for the entire network. In PaaS, the ZccN recharges both the selected node for recharge and other nodes around the selected node that requested recharge and has higher power transfer efficiency. This strategy of PaaS improves the charging efficiency of cloud chargers by minimizing the urgent charging requests in the future, and thus the operational efficiency of the MANET improves. Extensive simulations indicate that the proposed PaaS model with a hierarchy of cloud chargers improves the operational efficiency of MANETs in terms of reducing the death rate of mobile nodes, thereby improving the lifetime and connectivity probability of MANETs.
\end{abstract}

Keywords - Cloud-oriented approach; connectivity; Mobile Ad Hoc Networks; network lifetime; operational efficiency; Power-as-a-Service; Hierarchical Ondemand Charging Model 


\section{Introduction}

A Mobile Ad hoc Network (MANET) comprises a group of mobile nodes without any fixed infrastructure in which nodes communicate over multi-hop links. Military battlefields, post-disaster rescue efforts, sensor networks, and entrepreneurs in a conference are some of the examples of MANETs [1]. MANET typically consists of battery-dependent mobile nodes, which cooperate to transmit packets in the network. The existence of each node in the network is vital to impose such cooperation. Failure of any node in the network can affect the connectivity and lifetime of the entire network.

In MANETs, the limited power supply is a challenge. Because the MANET nodes are battery dependent, battery exhaustion is the primary reason for a node's failure. This problem has two solutions, either use energy-efficient strategies or wirelessly recharge the network nodes [2].

Existing energy-efficient strategies are classified into four categories [3]: (1) energy-efficient routing, (2) scheduling sleeping state of the nodes, (3) topology control by tuning node transmission power, and (4) limiting granularity of the information transferred. The best energy efficiency can be achieved by combining all the above four strategies. All these strategies have their advantages and disadvantages, and all insist on the constrained use of mobile nodes' battery energy.

Even though mobile nodes' lifetime can be prolonged via the above-mentioned energy-efficient strategies or with the use of powerful batteries, they can only defer the end of their battery life and could not provide nonstop functioning networks [4]. Recent works in the field of wireless energy transfer [5], [6], and rechargeable lithium batteries [7] initiated new trends in solving the battery depletion problem of mobile nodes.

On the other hand, today, cloud computing plays a vital role in the information technology industry. Cloud computing refers to services available over the Internet on-demand. Cloud resources can be easily scaled and rapidly provisioned regardless of the user location or device. Nowadays, cloud computing is not just a technology, and it is slowly transforming into an approach. An approach is a way of dealing with a situation or problem. To improve the operational efficiency of MANETS (i.e., the network lifetime and connectivity) as regards the battery power limitations of mobile nodes, this paper applies a "Cloud" oriented approach known as Power-as-a-Service (PaaS) to MANETs to overcome the limitations of battery-operated mobile nodes and to improve the operational efficiency by wirelessly recharging the batteries of the mobile nodes. We define a "Cloud" oriented approach to deal with a situation or a problem by applying the cloud service model's orientation. 
Mainly, our contributions in this paper include the following:

- MANET's overall operational efficiency is addressed in both connectivity of the network and nodes' lifetime. While the critical nodes (i.e., urgent charging requests) should always be served first, the critical nodes are given higher priority than other nodes' charging requests to benefit the network's overall operational efficiency.

- To obtain an overall operational efficiency of MANETs, a hierarchical charging model is proposed.

- To the best of our knowledge, we are the first to implement a "Cloud" oriented approach and a hierarchical charging model for improving the operational efficiency of MANETs.

- Scheduling algorithms for both the chargers in the hierarchical charging model are proposed.

- To demonstrate the performance of PaaS, extensive simulations are carried out, and the results are compared with the 'no charger case.

The remainder of this paper is organized as follows. Section 2 gives a brief overview of recent studies on on-demand charging in Wireless Sensor Networks (WSNs) and their PaaS differences. Section 3 presents the PaaS model and the charging schemes in detail. Section 4 presents performance evaluation and comparisons. Section 5 concludes this paper and outlines future work.

\section{Related Work}

Although current mobile nodes are built with more powerful batteries, their primary concern is still battery backup [8]. Some of the reasons why the batteries of mobile nodes drain very quickly include wireless communications, using numerous widgets, third party customizations, health trackers, having Wi-Fi turned on [8], using resource-intensive apps, content uploads, content backups, unnecessary push notifications, and apps are running in the background. Thus, there is a strong need to develop strategies that recharge present mobile nodes' batteries in MANETs.

To date, there is very little work done in the literature on recharging the batteries of mobile nodes of MANETs; as MANET is a general concept that can be implemented in sensors, laptops, and PC and its attachments [9] and most WSNs use the MANET model, here we review some of the state-of-the-art efficient on-demand wireless charging techniques of sensor nodes in WSNs.

P2S algorithm for recharging wireless sensor networks was suggested by Lin et al. [10]. P2S integrates primary charging with passer-by charging to reduce the number of emergency charging requests, improve the reliability, charging performance, and successful charging rate of wireless charging vehicles (WCV). P2S is effective in that it charges those nodes that are not urgently required by using the closeness of WCV to reduce the death rate and the sensor latency.

Lin et al. [11] proposed an on-demand Temporal-Spatial Real-Time Charging Scheduling Algorithm (TSCA) for Wireless Rechargeable Sensor Networks 
(WRSNs). TSCA maximizes the charging efficiency by minimizing the number of dead nodes to prolong the network lifetime. A feasible WCV movement solution is computed based on the gathered charging requests. Better efficiency is achieved by introducing a basic path planning algorithm that adjusts the charging order of WCV. Two algorithms are developed; one is a node deletion algorithm to remove lowefficiency charging nodes, and the other is a node insertion algorithm that avoids the death of abandoned nodes. Simulations are conducted to show that TSCA achieved promising performance in terms of charging throughput, charging efficiency, and other performance metrics compared to state-of-the-art charging scheduling algorithms.

Zhan et al. [12] suggested an effective rechargeable sensor network charging strategy. Their proposed strategy divides the sensor nodes of the service pools into two groups based on the on-demand sensor network's characteristics so that the mobile charger can offer to charge service priority as a matter of urgency during charging tour.

He et al. [13] proposed Nearest-Job-Next with Preemption (NJNP), an on-demand mobile charging discipline for the mobile charger. When a mobile nodes' residual energy falls below the threshold, it sends a charging request to the mobile charger. Then the mobile charger serves the requests received with NJNP. The mobile charger replenishes energy through an energy tank located at the center of the network. Extensive simulations are conducted to verify the accuracy and efficiency of NJNP.

The scheduling algorithm known as DWDP was introduced by Lin et al. [14] for WRSN on-demand charging. Two warning thresholds were established, and rules were compared to determine the timing priority for charge requests. Two preemption mechanisms were also designed to deal with WRSN's real-time features. The simulation results have shown that the DWDP is above the cutting-edge NJNP system concerning metrics such as charging rate, throughput, and charging success.

With the aid of several mobile wireless chargers, Wang et al. [15] considered the on-demand issue of charging for large sensor networks. Two methods were evaluated: recent-rarest-first and nearest-first. The simulation results showed that the recentrarest-first charger planning strategy matches the localized charging request framework while the nearest-first charger planning strategy matches the uniform charging request context.

Liang et al. [16] studied the problem of recharging wireless rechargeable sensor networks with an energy capacity constraint imposed on each mobile vehicle such that an optimum number of wireless mobile vehicles are used to recharge the sensors so that none of the sensors will drain out of energy. Experiments carried out show that the solution obtained is fractional of the optimal.

In [17], the authors examined energy transfer ideas briefly in WSN sensor nodes. They identified the latest developments in wireless technology to charge the batteries of sensor nodes on a WSN. A comparative survey of famous Wi-Fi recharging techniques for charging sensor nodes in WSN was provided. They suggested an approach where the sensor network was split up into two clusters, and each cluster had a distinct charging vehicle; the principle of energy consciousness was decided on the traveling trajectory of the charging vehicle; and the direction on which the energy node 
was also installed from the point of departure of the wireless charging vehicle. To minimize charging time and complexity, the proposed method improved efficiency.

A detailed peer-to-peer energy sharing survey was carried out in [18] in four separate implementations of mobile networks: wireless sensor networks (WSN), vehicular ad hoc networks (VANET), mobile social networks (MSN), and UAV networks. The authors studied the use of energy sharing for each application, what types of issues are addressed, and what technological methods and mathematical instruments are adopted.

The authors in [19] proposed a common spatial correlation transmission of data and an on-demand algorithm for charging. The proposed algorithm optimized event detection, data transmission, and node charging together to increase charging speed. First, an enhanced iterative node selection approach will be taken to reduce the number of nodes that operate at a time to select active nodes that participate in the event detection. The greedy grid-based framework for transmitting the data observed to the sink node is proposed. Finally, the network nodes are charged using the grid-based on-demand charging system, which significantly reduces the mobile charger's charging frequency and power loss. The simulation results show that the proposed approach is performing more highly in the distance of the mobile charger, energy consumption, average energy consumption, and node charging latency.

The use of energy sharing in the domain wireless sensor networks has been extensively studied in the literature, but in other fields such as MANETs, it is also a very active field of study. The noticeable difference between the state-of-the-art and the proposed PaaS is that PaaS works on MANETs, whereas all the above works were conducted on WSNs. Most of the above works focused on prolonging the network lifetime, whereas our work considers both on prolonging the network lifetime and improving network connectivity by recharging the mobile nodes on-demand. Our work is quite different from the above works because they consider only one charging vehicle or multiple, whereas our work considers a hierarchy of charging vehicles.

The proposed approach maximizes the nodes' lifetime and minimizes node failures caused due to battery drains by dividing the MANET into zones and deploying a $\mathrm{ZccN}$ for every zone. This makes the proposed approach suitable for large-scale MANETs.

To the best of our knowledge, our work is the first attempt to improve the operational efficiency of MANETs in terms of lifetime and connectivity by considering a "Cloud" oriented approach with a hierarchy of chargers.

\section{The PaaS Model}

The proposed PaaS model features three types of devices: regular mobile nodes, Zone Charging Cloud Nodes (ZccNs), and High-power Charging Cloud Nodes (HccNs). The notations used in this paper are summarized in Table 1. 
Table 1. Symbols and Definitions

\begin{tabular}{|c|l|}
\hline Symbol & \multicolumn{1}{|c|}{ Definition } \\
\hline $\mathrm{M}$ & Mobile Ad hoc Network. \\
\hline $\mathrm{Z}$ & Zones in a MANET, $\mathrm{Z}=\left\{\mathrm{Z}_{1}, \mathrm{Z}_{2}, \ldots\right\}$. \\
\hline $\mathrm{N}$ & Mobile nodes deployed in a zone, $\mathrm{N}=\left\{\mathrm{N}_{1}, \mathrm{~N}_{2}, \ldots\right\}$. \\
\hline $\mathrm{ZccN}$ & Zone Charging Cloud Node. \\
\hline $\mathrm{M}_{\mathrm{Z}}$ & Number of ZccNs in $\mathrm{M}$. \\
\hline $\mathrm{HccN}$ & High-power Charging Cloud Node. \\
\hline $\mathrm{NE}_{\max }$ & The maximum battery capacity of mobile node $\mathrm{N}_{\mathrm{i} .}$ \\
\hline $\mathrm{NE}_{\min }$ & The minimum level of battery required by mobile node $\mathrm{N}_{\mathrm{i}}$ for it to be operational. \\
\hline $\mathrm{NE}_{\mathrm{th}}$ & Energy threshold of mobile node $\mathrm{N}_{\mathrm{i}}$ to make a recharge request. \\
\hline $\mathrm{RE}_{\mathrm{n}}$ & The residual energy of mobile node $\mathrm{N}_{\mathrm{i}}$. \\
\hline $\mathrm{ZE}_{\max }$ & The maximum battery capacity of a $\mathrm{ZccN} \mathrm{Z}_{\mathrm{i}}$. \\
\hline $\mathrm{ZE}_{\min }$ & The minimum level of battery required by a $\mathrm{Z}_{\mathrm{i}}$ for it to be operational. \\
\hline $\mathrm{ZE}_{\mathrm{th}}$ & Energy threshold of a $\mathrm{Z}_{\mathrm{i}}$ to make a recharge request. \\
\hline $\mathrm{RE}_{\mathrm{z}}$ & The residual energy of a $\mathrm{Z}_{\mathrm{i}}$. \\
\hline $\mathrm{HE}_{\max }$ & The maximum battery capacity of a HccN. \\
\hline $\mathrm{HE}_{\mathrm{th}}$ & Energy threshold of HccN to move back to the home service station to get recharged. \\
\hline $\mathrm{RE}_{\mathrm{h}}$ & The residual energy of HccN. \\
\hline
\end{tabular}

The MANET is divided into $\mathrm{Z}$ non-overlapping disjoint zones. Each zone $\mathrm{Zi}$ (i=1 to $\mathrm{n}$ ) contains $\mathrm{N}$ mobile nodes. The nodes in the network are equipped with Global Positioning System (GPS). Nodes identify the current location and obtain their respective Zone IDs using GPS. Mobile nodes are assumed to move and perform their assigned tasks within the deployed area. Each mobile node $\mathrm{Ni}(\mathrm{i}=1$ to $\mathrm{n}$ ) has a battery with a capacity of NEmax and is fully charged initially. Also, we denote NEmin as the minimum energy level at a battery for the mobile node to be operational.

\subsection{Basic idea of PaaS}

The proposed PaaS model is a hierarchical charging model in which the $\mathrm{HccN}$ is the highest device in terms of the hierarchy and can charge the ZccNs, which can charge the mobile nodes. Thus, a $\mathrm{HccN}$ is responsible for recharging the $\mathrm{ZccNs}$, and the $\mathrm{ZccNs}$ are responsible for recharging the mobile nodes.

To wirelessly recharge the battery of the regular mobile nodes in each zone, a $\mathrm{ZccN}$ is employed. To recharge the batteries of $\mathrm{ZccNs}$, an $\mathrm{HccN}$ is employed within the network. In the PaaS model, we assume that one $\mathrm{ZccN}$ and the entire network for each zone, one $\mathrm{HccN}$ is sufficient to recharge regular nodes and $\mathrm{ZccNs}$, respectively. Further, multiple mobile nodes are charged simultaneously by the ZccN.

\subsection{On-demand charging with ZccNs}

The lifetime of the network is known as the time when energy runs out of the first node. Network connectivity measures the degree and the ease (speed) to which the network components (nodes) are linked. A node in the network whose failure causes the network to be partitioned into two or more separate components is known as a 
critical node [20], [21]. Failure of a critical node significantly degrades the performance of the network by introducing unacceptable long alternative paths. The death of a node in the network will significantly influence the network lifetime and connectivity. Recharging a node can significantly increase the network lifetime and connectivity only when it is about to drain out of energy. This strategy of recharging is known as "on-demand recharging" [22].

$\mathrm{ZccN}$ follows the on-demand recharging strategy because the proposed PaaS is a "Cloud" oriented approach, in which the services (recharging) are provided ondemand to the clients (mobile nodes). With GPS's help, ZccN identifies mobile nodes' locations and uses this information to calculate the route. Usually, a ZccN starts from one end of the zone, follows a specific predefined route [23], and uses long-range radio waves [24] to perform a periodical broadcast of its location nodes in its zone.

When the REn of Ni falls below the NEth $\in$ (NEmin $>$ NEth<NEmax), Ni will send a recharging request to the $\mathrm{ZccN}$ in its zone for energy replenishment; the request contains the node's identity and location parameters. On receiving charging requests, the decision as to which nodes are to be recharged is made by the ZccN by the following charging policies.

\subsection{ZccN charging policies}

- Policy 1 (P1) considers charging of the critical node and other nodes around the critical node that requested energy charging and has higher power transfer efficiency. If there is more than one critical node in the zone, they are recharged on a FirstRequest-First-Service (FRFS) basis.

- Policy2 (P2) If there are no critical nodes among the nodes that requested charging, then the ZccN charges the nodes on an FRFS basis and other nodes around the selected node that requested energy charging and have higher power transfer efficiency towards them.

Depending on the specific charging policy, some nodes may be preferred over other nodes. Hence, the nodes that are to be charged by each ZccN in each zone may change depending on the policy adopted by the ZccN.

Irrespective of the policy selected by the $\mathrm{ZccN}$, a certain amount of power will be wirelessly transmitted to the selected nodes by the ZccN. If policy P1 is selected, then the amount of energy transmitted to the critical node will be transmitted to the other nodes. If policy P2 is selected, then the amount of energy transmitted to the selected node as per FRFS will be compared to the other nodes. The number of time slots [25] allocated to the selected node as per FRFS will be more than to the other nodes. Therefore, the amount of energy that is to be transmitted by $\mathrm{ZccN}$ to each node may vary. After recharging, the ZccN moves back to its original route; if there are no more recharging requests, if there are more recharging requests, then the ZccN moves directly to the node that has to be recharged. The algorithm for $\mathrm{ZccN}$ operations is given in the section 3.4. 


\subsection{Algorithm for ZccN operation}

Algorithm 1. ZccN Operation

Input

$\mathrm{N}$ : number of Mobile Nodes in zone $Z_{i}$

$\mathrm{R}$ : the $\mathrm{ZcCN}$ route

\section{Output:}

$\mathrm{N}_{i}$ is Recharged

Other nodes around $\mathrm{N}_{i}$ that requested energy charging and have higher power transfer efficiency towards them are also recharged

1. ZccN moves on a route $R$

2. ZccN broadcasts location messages periodically

3. $\mathrm{N}_{i}$ sends a charging request to $\mathrm{ZCCN}$ when $\mathrm{RE}$

$\left(\mathrm{N}_{i}\right)<=\mathrm{NE}_{\mathrm{th}}$

4. On reception of a charging request from $\mathrm{N}_{i}$

4.1. ZccN performs Critical Node Test

4.2. if $\mathrm{N}_{i}$ is a Critical Node, then ZccN sends a Reply message to node $\mathrm{N}_{i}$

ZccN calculates a temporary route (TR) to meet node $\mathrm{N}_{i}$ On the ZccN meeting node $\mathrm{N}_{i}$

Apply Policy-1

else

ZccN sends a Reply message to node $\mathrm{N}_{i}$ ZccN calculates a temporary route TR to meet node

$\mathrm{N}_{\mathrm{i}}$

On the ZccN meeting node $\mathrm{N}_{i}$

Apply Policy-2

endif

5. On completion of recharging

5.1. if further recharge requests exist, then

ZccN moves to reach the next selected node that has to be recharged

else

ZccN moves back to its route $R$

ZcCN moves forward on its route $R$

endif

\subsection{On-demand charging with HecN}

Using GPS, HccN identifies the list of ZccNs in the network and uses this information for calculating the route. Usually, the HccN starts from a home station, fol- 
lows a specific predefined route [23], and uses long-range radio waves [24] to perform a periodical broadcast of its location ZccNs. When the REz of a ZccN falls below the ZEth $\in(Z E m i n>Z E t h<Z E m a x)$, the $Z c c N$ will send a recharging request to the $\mathrm{HccN}$ for energy replenishment, with the request containing its identity and location parameters.

Upon reception of a request message, the $\mathrm{HccN}$ adjusts its route to meet the $\mathrm{ZccN}$ and recharge the ZccN's battery. The HccN charges each ZccN to the maximum level. After completion of recharging, the $\mathrm{HccN}$ moves back to its original route if there are no outstanding recharging requests; if there are any recharging requests, the $\mathrm{HccN}$ moves directly to the $\mathrm{ZccN}$ that has to be recharged. Each $\mathrm{HccN}$ charges the ZccNs on an FRFS basis. When the REh energy falls below HEth, the HccN visits the home service station, gets recharged, and restarts its journey. The algorithm for HccN operations is given in section 3.6.

\subsection{Algorithm for $\mathrm{HecN}$ operation}

\section{Algorithm 2. HccN Operation}

\section{Input}

$\mathrm{M}_{\mathrm{Z}}$ : number of $\mathrm{ZCCNS}$ in $\mathrm{M}$

$\mathrm{R}$ : the $\mathrm{HCCN}$ route

\section{Output:}

$Z_{i}$ is recharged

1. HCCN moves on a route $\mathrm{R}$

2. HCCN broadcasts location messages periodically

3. $Z_{i}$ sends a charging request to $\operatorname{HccN}$ when $\operatorname{RE}\left(Z_{i}\right)<=$ ZEth

4. On reception of a charging request from $Z_{i}$ HCCN selects a $Z_{i}$ based FRFS

5. HccN sends a Reply message to the selected Zi

6. HCcN calculates a temporary route $T R$ to meet the $Z_{i}$

7. On HccN meeting $Z_{i}$

HccN recharges $Z_{i}$ to the maximum level

8. On completion of recharging

if further recharge requests exist, then

HcCN moves to reach the next selected $Z_{i}$ that

has to be recharged

\section{else}

HCCN moves back to its route $R$

HCCN moves forward on its route $R$

endif 


\section{Performance Evaluation}

In this section, we evaluate the PaaS approach concerning the no-charger case (absence of PaaS) in terms of the following performance metrics:

- Alive nodes over time: The number of nodes with enough residual energy to operate during the experiment's progress.

- Connectivity probability: A measure of the time intervals during which the network is connected.

To demonstrate the performance of PaaS, extensive simulations are conducted through MATLAB R2017a. The simulation parameters were primarily chosen based on [11]. All the regular nodes, ZccNs, and $\mathrm{HccN}$, were fully charged before starting the simulation. Specific simulation parameters and their values are listed in Table 2.

Table 2. Simulation Parameters

\begin{tabular}{|l|c|}
\hline \multicolumn{1}{|c|}{ Symbol } & Definition \\
\hline Network size & $500 \times 500 \mathrm{~m}$ \\
\hline Number of nodes & 80 \\
\hline The energy consumption rate of node & $0.06-0.11 \mathrm{~J} / \mathrm{s}$ \\
\hline Threshold for node & $30 \%$ \\
\hline Threshold for ZccN & $35 \%$ \\
\hline Simulation time & $600 \mathrm{~s}$ \\
\hline
\end{tabular}

The overall death rate (in terms of alive nodes over time) of the network was substantially reduced, as shown in Fig. 1. Fig. 3 depicts the percentage of alive nodes zone-wise in the MANET. The figure shows that more than 50\% of nodes are still alive in the network. With this observation, it is clear that there is a substantial improvement in the number of alive nodes in the network with the PaaS approach. This reduction in the overall death rate of mobile nodes improves the overall network lifetime.

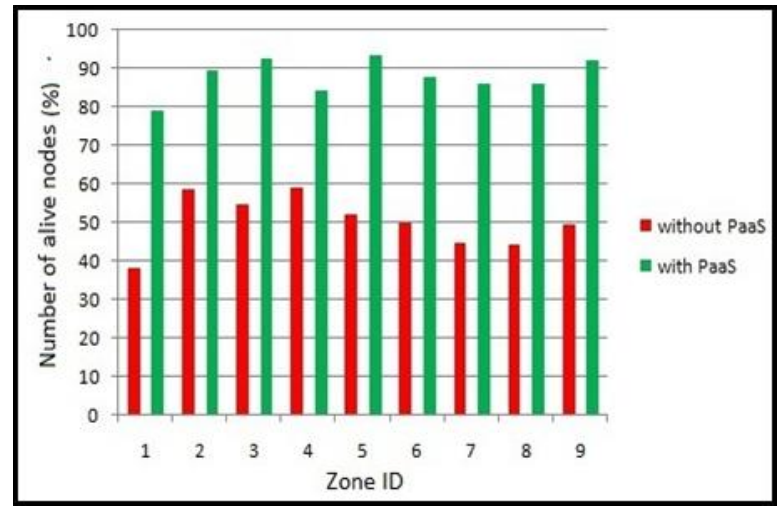

Fig. 1. The average number of alive nodes in each zone 
The average connectivity probability of the network over time is depicted in Fig. 4. From the Fig. 4, it is observed that the probability of having a connected network is improved about $50 \%$ with the application of the PaaS approach.

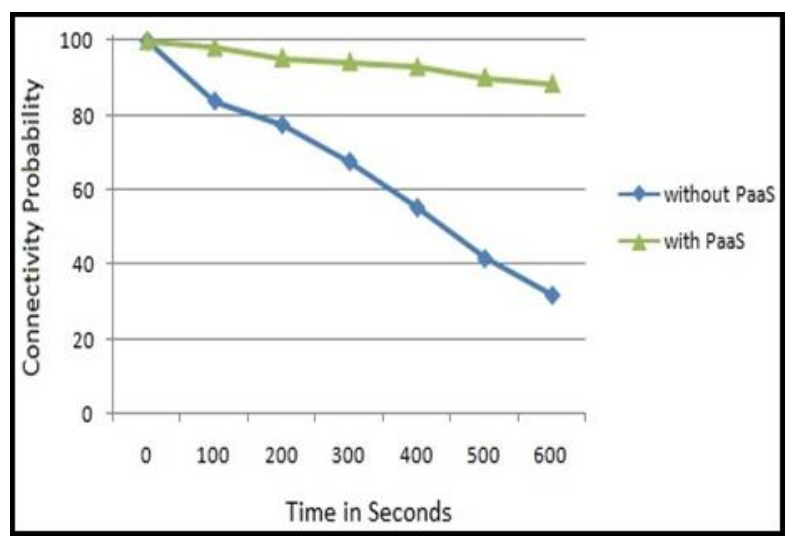

Fig. 2. Connectivity probability of the Network

The simulation results demonstrate significant performance gains, both concerning the number of alive nodes and the network's connectivity with the PaaS approach.

\section{$5 \quad$ Conclusion and Future Work}

Motivated by the recent trends in the wide range of cloud models' application scenarios, we proposed a Power-as-a-Service (PaaS) approach to improve the operational efficiency of MANETs. PaaS minimizes the number of dead nodes by recharging mobile nodes on-demand while maximizing the network lifetime and connectivity. In PaaS, a ZccN recharges both the selected node for recharge and other nearby nodes around the selected node that are requested for recharge and have higher power transfer efficiency. Such a combined strategy improves cloud chargers' charging efficiency by reducing future urgent charging requests. Furthermore, the results of extensive simulations conducted show that compared with the no-charger case PaaS achieves promising performance in terms of connectivity probability and alive nodes over time, thereby improving the operational efficiency of MANETs.

As part of our future work, we will focus on the collaboration of $\mathrm{ZccN}$ and $\mathrm{HccN}$ for charging critical nodes, the recharging strategy of the $\mathrm{HccN}$, deployment of multiple HccNs, failure of ZccNs, and HccNs, and optimal placement of charging stations for HccNs.

\section{$6 \quad$ References}

[1] K. Muralidhar and K. Madhavi, "An investigation into the operational limitations of mobile ad hoc networks," presented at the 2017 International Conference on Wireless Com- 
munications, Signal Processing and Networking, Chennai, India, Mar. 22-24, 2017. https://doi.org/10.1109/wispnet.2017.8299988

[2] M. Bhardwaj," Enhance life time of mobile ad-hoc network using WiTriCity and backpressure technique," Procedia Computer Science, vol. 57, pp. 1342-1350, 2015. https://doi.org/10.1016/j.procs.2015.07.447

[3] S. Mahfoudh and P. Minet, "Survey of energy efficient strategies in wireless ad hoc and sensor networks," in Proc. Seventh International Conference on Networking, Cancun, 2008, pp. 1-7. https://doi.org/10.1109/icn.2008.55

[4] E. Bulut, M. E. Ahsen and B. K. Szymanski, "Opportunistic wireless charging for mobile social and sensor networks," in Proc. 2014 IEEE Globecom Workshops, 2014, pp. 207212. https://doi.org/10.1109/glocomw.2014.7063432

[5] A. Kurs, A. Karalis, R. Moffatt, J. D. Joannopoulos, P. Fisher and M. Soljacic, "Wireless power transfer via strongly coupled magnetic resonances," Science, vol. 317, pp. 83-86, July 2007. https://doi.org/10.1126/science.1143254

[6] B. Cannon, J. Hoburg, D. Stancil and S. Goldstein, "Magnetic resonant coupling as a potential means for wireless power transfer to multiple small receivers," IEEE Trans. on Power Electronics, vol. 24, no. 7, pp. 1819-1825, July 2009. https://doi.org/10.1109/tpe 1.2009 .2017195

[7] K. Kang, Y. S. Meng, J. Breger, C. P. Grey and G. Ceder, "Electrodes with high power and high capacity for rechargeable lithium batteries," Science, vol. 311, pp. 977-980, Feb. 2006. https://doi.org/10.1126/science. 1122152

[8] A. Prabhu, "6 reasons your smartphone battery is draining quickly," https://www.gizbot. com/mobile/features/smartphone-battery-life-drain-reasons-resolve-issues-040240.html, $\underline{2017}$.

[9] A. Umar, "Mobile computing and wireless communications," NGE Solutions, 2004, pp. 10-18.

[10] C. Lin, D. Han, J. Deng and G. Wu, "P2S: A primary and passer-by scheduling algorithm for on-demand charging architecture in wireless rechargeable sensor networks," IEEE Transactions on Vehicular Technology, Vol. 66, no. 9, pp. 8047-8058, Sept. 2017. https://doi.org/10.1109/tvt.2017.2683493

[11] C. Lin, J. Zhou, C. Guo, H. Song, G. Wu and M. S. Obaidat, "TSCA: A temporal-spatial real-time charging scheduling algorithm for on-demand architecture in wireless rechargeable sensor networks," IEEE Transactions on Mobile Computing, vol. PP, no.99, pp.1-14, 2017. https://doi.org/10.1109/tmc.2017.2703094

[12] S. Zhan, J. Wu, L. Qu and D. Xin, "Efficient scheduling strategy for mobile charger in wireless rechargeable sensor networks," in Proc. PDCAT, Guangzhou, 2016, pp. 36-39. https://doi.org/10.1109/pdcat.2016.023

[13] L. He, L. Kong, Y. Gu, J. Pan and T. Zhu, "Evaluating the on-demand mobile charging in wireless sensor networks," IEEE Transactions on Mobile Computing, vol. 14, no. 9, pp. 1861-1875, Sept. 2015. https://doi.org/10.1109/tmc.2014.2368557

[14] C. Lin, B. Xue, Z. Wang, D. Han, J. Deng and G. Wu, "DWDP: A double warning thresholds with double preemptive scheduling scheme for wireless rechargeable sensor networks," in Proc. 2015 HPCC, 2015 IEEE CSS, and 2015 IEEE ICESS, New York, NY, 2015, pp. 503-508. https://doi.org/10.1109/hpcc-css-icess.2015.115

[15] J. Wang, T. Si, X. Wu, X. Hu and Y. Yang, "Sustaining a perpetual wireless sensor network by multiple on-demand mobile wireless chargers," in Proc 2015 IEEE 12th International Conference on Networking, Sensing and Control, Taipei, 2015, pp. 533-538. https://doi.org/10.1109/icnsc.2015.7116093 
[16] W. Liang, W. Xu, X. Ren, X. Jia and X. Lin, "Maintaining sensor networks perpetually via wireless recharging mobile vehicles," in Proc. 39th Annual IEEE Conference on Local Computer Networks, Edmonton, AB, 2014, pp. 270-278. https://doi.org/10.1109/lcn.201 4.6925781

[17] Prakash S., Saroj V., "A Review of Wireless Charging Nodes in Wireless Sensor Networks," In: Mishra D., Yang XS., Unal A. (eds) Data Science and Big Data Analytics. Lecture Notes on Data Engineering and Communications Technologies, vol 16, 2019, Springer, Singapore. https://doi.org/10.1007/978-981-10-7641-1_15

[18] Aashish Dhungana, Eyuphan Bulut, "Peer-to-peer energy sharing in mobile networks: Applications, challenges, and open problems," Ad Hoc Networks, Vol. 97, 2020, pp. 1-16. https://doi.org/10.1016/j.adhoc.2019.102029.

[19] Hu Q, Yang J, Huo Y, Li B, Li S., "Space-correlation-based joint data transmission and on-demand charging for rechargeable wireless sensor networks," IET Commun., vol. 15, 2021, pp. 546-557. https://doi.org/10.1049/cmu2.12086

[20] M. Sheng, J. Li and Y. Shi, "Critical nodes detection in mobile ad hoc network," in Proc. AINA'06, Austria, 2006, pp. 5.

[21] M. Jorgic, I. Stojmenovic, M. Hauspie and D. Simplot-Ry, "Localized algorithms for detection of critical nodes and links for connectivity in ad hoc networks," in Proc. 3rd IFIP Mediterranean Ad Hoc Networking Workshop, Procedia Computer Science, vol. 19, pp.1167-1172, 2013. https://doi.org/10.1016/j.procs.2013.06.166

[22] N. Meghanathan and A. Farago, "Maximizing network lifetime under a fixed energy budget in mobile ad hoc networks," in Proc. IEEE SoutheastCon, 2005, pp. 319-326. https://doi.org/10.1109/secon.2005.1423266

[23] C. Qin, Y. Sun, Y. Zhang and M. Ai, "A novel path planning of mobile charger in wireless rechargeable sensor networks," in Proc. 29th Chinese Control and Decision Conference (CCDC), Chongqing, 2017, pp. 2063-2067. https://doi.org/10.1109/ccdc.2017.7978856

[24] K. Muralidhar and N. P. Bharathi, "Prolonging the MANET lifetime through wireless energy transfer using high powered and native powered mobile nodes," in Proc. ICAECC, Bangalore, 2014, pp. 1-4. https://doi.org/10.1109/icaecc.2014.7002418

[25] L. Bonati, Á. F. Gambín and M. Rossi, "Wireless power transfer under the spotlight: Charging terminals amid dense cellular networks," in Proc. 2017 IEEE WoWMoM, Macau, 2017, pp. 1-9. https://doi.org/10.1109/wowmom.2017.7974284

\section{Authors}

Mr. Muralidhar Kurni is an Independent Consultant for Pedagogy Refinement, EduRefine, India. He is currently working as an Assistant Professor in the Department of Computer Science \& Engineering at Anantha Lakshmi Institute of Technology \& Sciences, Anantapuramu, Andhra Pradesh, India. Previously he worked as Head, Department of Computer Applications, Sri Sai College of Technology \& Management, Kadapa, Andhra Pradesh, India. Mr. Muralidhar Kurni has received his M.Sc. in Computer Science from S. K. University and M.Tech. in Computer Science \& Engineering from JNTUA, Ananthapuram, Andhra Pradesh, India. He has more than 19 years of teaching experience. He is an IUCEE \& IGIP certified International Engineering Educator \& researcher. He has several scholarly publications to his credit. He presented about 30 papers at various national and international conferences and journals. Four of his papers received the best paper awards. Two of them are the IEEE 
Conference Best Paper awards. He has been a reviewer for various International Conferences \& Journals, including SCIE \& Scopus indexed Journals. He served as a Guest Editor for the Special Issue on "Security, Privacy, and Trust in IoT" of IJWNBT Journal, IGI Global, Volume 8, Issue 2, July-December 2019. His research interests include Mobile Ad Hoc Networks, Cloud Computing, Ad Hoc Mobile Cloud Computing, Learning Analytics, Learning Strategies, Digital Pedagogy, Design Thinking, Pedagogy refinement \& Engineering Education Research.

Dr. K. Saritha is currently working as Principal, S.V. Degree \& P.G. College, Anantapur, Andhra Pradesh. She worked as Assistant Professor \& Skill Development Co-ordinator at Madanapalle institute of Technology \& Science, Madanapalle, Chittoor and worked as Assistant Professor at Dept. of MCA, K.S.R.M College of Engineering, Kadapa, Andhra Pradesh and Lecturer at Computer Science department, National Degree College, Nandyal, Andhra Pradesh. She has conducted several skill development activities for students through APSSDC at Madanapalle Institute of Technology \& Science and two of the student apps got selected as best apps by Andhra Pradesh State Government. She has received M.Sc Computer Science from S.K. University, Anantapur, Andhra Pradesh, M.Phil in Computer Science from Sri Padmavati Mahila Visvavidyalayam (Women's University), Tirupati, Andhra Pradesh and Ph.D in Computer Science from Sri Padmavati Mahila Visvavidyalayam (Women's University), Tirupati, Andhra Pradesh, India. She has 20+ years teaching experience. She has Presented15 papers at various national \& international conferences and journals. Her research interests are Data Mining, Blockchain, Cloud Computing, Machine Learning.

Article submitted 2021-04-03. Resubmitted 2021-05-25. Final acceptance 2021-05-26. Final version published as submitted by the authors. 